\title{
E-Health in der Psychiatrie
}

\author{
E-Health in Psychiatry
}

Autoren

Institut
Michael Lucht, Georg Schomerus

Klinik und Poliklinik für Psychiatrie und Psychotherapie der Universitätsmedizin Greifswald am HELIOS-Hanseklinikum Stralsund

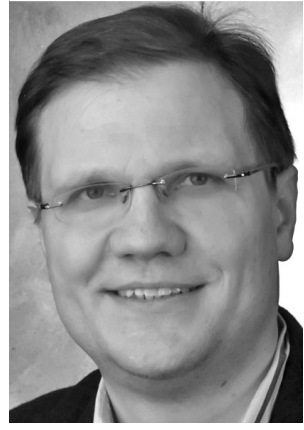

Michael Lucht

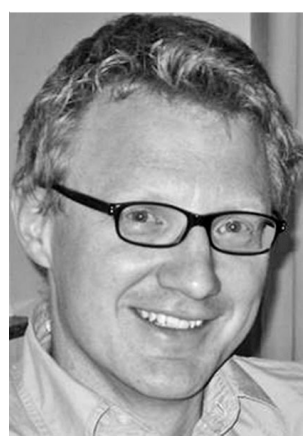

Georg Schomerus

Bibliografie

Dol http://dx.doi.org/

10.1055/s-0033-1349424

Psychiat Prax 2013; 40:

301-303

(c) Georg Thieme Verlag KG Stuttgart · New York

ISSN 0303-4259

Korrespondenzadresse Prof. Dr. med. Michael Lucht Klinik und Poliklinik für Psychiatrie und Psychotherapie der Universitätsmedizin Greifswald am HELIOSHanseklinikum Stralsund Rostocker Chaussee 70 18435 Stralsund lucht@uni-greifswald.de
Medizinische Behandlung über lange Distanzen ist seit Jahrzehnten Gegenstand faszinierender Berichte: aus entlegenen Gebieten Australiens, aus der Raumfahrt, von Schiffen oder Arktisstationen wurden immer wieder spektakuläre Aktionen berichtet. Es ist inzwischen möglich, EKGs, hochauflösende radiologische oder histologische Bilder zu übermitteln und danach Therapieentscheidungen zu treffen. Das spart Ressourcen und macht Kompetenz weit verfügbar.

\section{Nicht nur Kommunikationsmittel, sondern Therapieinstrument \\ $\nabla$}

E-Health-Ansätze mit Telefon, SMS, Internet, Apps oder Video-Konferenzen zur Behandlung psychischer Störungen überbrücken im Vergleich mit somatischen Störungen nicht nur Entfernungen, sondern ermöglichen ganz neue therapeutische Strategien [1, 2]. Elemente sind die regelmäßige Erfassung von Zustandsveränderungen, Symptomen und Hilfebedarfen (Surveillance), angeleitete Selbsthilfe-Interventionen, therapeutische Dialoge, Selbsthilfe-Foren, Anleitung zu Skills bzw. die Möglichkeit, Notrufe abzusetzen. E-Health-Programme können vollautomatisch (Raucherbehandlungssystem txt2stop, [3]) oder mit Therapeutenbeteiligung (Expertensystem für Raucherentwöhnung in der Hausarztpraxis [4]) betrieben werden. Es ist möglich, dass so tausende von Patienten zu bisher nie gekannt niedrigen Kosten teilnehmen können (weitere Beispiele: SUMMIT-Internetstudie bei chronischer Depression, MoodGym und Deprexis: Online-Programm gegen Depressionen, Lebenstagebuch: Schreibtherapie für traumatisierte Kriegsüberlebende des Zweiten Weltkriegs, ProYouth: Online-Programm bei Essstörungen).

Ein Beispiel für ein einfaches Surveillance-System mit Therapeutenbeteiligung und unterstützendem Feedback ist CAPS: hier wird regelmäßig au- tomatisiert der Hilfebedarf von Patienten mit Alkoholabhängigkeit auf ihrem Handy abgefragt. Der Therapeut wird über E-Mail benachrichtigt, wenn der Patient einen Unterstützungswunsch „simst“, dann kann telefonisch ein Hilfsangebot durch den Therapeuten erfolgen (http://www. controlled-trials.com/isrctn/pf/78350716).

\section{Therapeutische Beziehungen wirken auch über Entfernungen $\nabla$}

Psychiatrische E-Health-Angebote machen sich darüber hinaus zunutze, dass psychosoziale Interventionen und Bindungen auch über Distanz wirken [5], und zwar sowohl mit als auch ohne Therapeut. Wie sonst wäre die Attraktivität der riesigen sozialen Netzwerke zu erklären, allein um Informationsübertragung kann es sich dabei nicht handeln. Es ist hochwahrscheinlich, dass neben spezifischen Interventionen unspezifische (aber kunstvolle) Wirkfaktoren nach Wampold wie (a) die Welt verstehen, (b) Veränderung durch soziale Mittel, (c) Verbundenheit, (d) Hoffnung und Bewältigung [6] auch bei E-Health-Interventionen wirksam sind.

Automatisierte E-Health-Systeme sind ein Motor zur Aufrechterhaltung von Kontakt. Dabei kann E-Health natürlich kein Ersatz für eine unmittelbare Begegnung sein. Aber die Einschränkungen der Kontaktqualität haben auch spezifische Vorteile: für Menschen mit sozialen Ängsten und Vermeidung werden so vielleicht sogar Kontakte leichter hergestellt, da eine gewisse soziale Distanz erhalten wird. Der niederschwellige Zugang, die zeitliche und örtliche Flexibilität, das selbstgewählte Tempo und die reduzierte wahrgenommene Stigmatisierung [7] sind Eigenschaften, die auch die neue S3-Leitlinie Psychosoziale Therapien bei schweren psychischen Erkrankungen [8] als positive Eigenschaften von E-Health hervorhebt. Es gibt gute Gründe anzunehmen, dass Un- 
gleichheit Ursache von psychischer Krankheit ist [9]. Das drückt sich insbesondere im sog. inverse care law aus [10,11], wonach Schwerkranke in der Psychiatrie häufig von den aufwendigen (Psycho-)Therapien ausgeschlossen sind. Hier könnte E-Health eine Hilfe sein, da auch für Patienten mit schweren Störungen und für sozial Benachteiligte einfache help-lines über Handy nutzbar sein könnten.

E-Health ist eine neue Chance für chronische psychische Störungen, gleichermaßen für z.B. Depression, Schizophrenie, Abhängigkeitserkrankungen. Das in der somatischen Medizin verbreitete Konzept der kontinuierlichen Behandlung ist auch ein gutes Modell für die Behandlung psychischer Störungen [12]. Ein Langzeitprogramm mit täglichen Kontakten zur Behandlung von Alkoholabhängigkeit zeigte sehr gute Ergebnisse [13], es hat aber vermutlich des Aufwands und der Kosten wegen nicht die ihm zukommende Verbreitung gefunden. Regelmäßige kleine Anstöße zur Veränderung sind mitunter wirksamer als ein großer Ansatz mit Heilungsanspruch. In der Suchtmedizin zeigt sich, dass selbst gering anmutende Trinkmengensenkungen zu einem großen Gewinn an Gesundheit führen [14]. Solche kontinuierlichen supportiven Monitoringkonzepte kann erst E-Health überhaupt in großer Zahl in die Fläche bringen. Kontinuierliches Monitoring ohne Therapeut markiert damit den diesseitigen Endpunkt des Therapiespektrums, Tilmann Moser zitiert Leon Wurmser im Ärzteblatt, wonach bei manchen Patienten erst ab $1450 \mathrm{~h}$ Veränderungen sichtbar werden [15]. E-Health wird nicht die Effekte und die Zuwendung bewirken, die Psychotherapie bringt, aber es ist wohl ausgeschlossen, dass für alle behandlungsbedürftigen Patienten jemals genug Therapeuten zur Verfügung stehen.

\section{E-Health kann existierende Strukturen in Versorgung und Forschung ergänzen und bereichern \\ $\nabla$}

Die Kombinationsmöglichkeiten sind beliebig: E-Health ist für Suchtberatung ebenso geeignet wie als add-on bei einer Psychotherapie; für Pharmakotherapie der Schizophrenie, zusammen mit Genotypisierung, TDM, Frühsymptommonitoring und NWManagement. E-Health kann hier ein psychosoziales Element individualisierter Medizin werden. E-Health-Monitoring erlaubt stepped-care als Taktgeber und einen gestuften Einsatz von Interventionen je nach Aufwand.

In ländlichen Gebieten wie z.B. Teilen Mecklenburg-Vorpommerns gibt es Regionen, in denen es nicht möglich ist, innerhalb eines Tages einen Psychotherapeuten mit öffentlichen Verkehrsmitteln zu erreichen und wieder nach Hause zu kommen. Im Rahmen der SHIP-Studie konnte gezeigt werden, dass in diesen Regionen die Mehrheit der Menschen mit einer schweren psychischen Störung unbehandelt sind. Die Frage nach einer alternativen Versorgung stellt sich hier unter den gegebenen Rahmenbedingungen gar nicht, hier könnte E-Health sogar erstrangige Behandlung werden.

Im Straßenverkehr hat v.a. die weite Verbreitung von Technik (Knautschzone, Airbag und die Entschärfung von Unfallschwerpunkten) die Unfallzahlen drastisch reduziert, und nicht eine eventuelle Veränderung von Einstellungen oder Verhalten. Auch Telemedizin ist eine Technik mit dem Potenzial weiter Verbreitung. Sie kann Menschen helfen, denen eine dauerhafte Veränderung ihres Gesundheitsverhaltens zunächst nur schwer möglich ist, die aber von kontinuierlicher Unterstützung profitieren. Ein Beispiel wäre ein Notruf per SMS bei Zunahme psychotischer
Symptomatik. Verhaltensänderungen sind auch hierbei natürlich sekundär möglich. Allerdings bedarf es hierzu Anbieter, die EHealth weit verbreiten und verfügbar machen würden.

Interaktive telemedizinbasierte Ansätze eröffnen eine neue Chance für direkte und v. a. lokale Evaluation in der Versorgungsforschung. E-Health-Applikationen produzieren nämlich immer ihren eigenen Datensatz an Real-time-Indikatoren. Die Güte der in E-Health gewonnenen Indikatoren scheint ausreichend $\mathrm{zu}$ sein: so unterschieden sich E-Health-Daten zur Trinkmenge über SMS [16] nicht wesentlich von katamnestisch gewonnenen Werten. Für viele Therapieentscheidungen ist eine genauere Information vermutlich gar nicht notwendig.

\section{Der Mensch und sein Datensatz dürfen nicht verwechselt werden \\ $\nabla$}

Die Aussichten klingen überaus positiv. Aber gibt es auch Risiken und Nebenwirkungen? Wir möchten sagen: sogar erhebliche. Die Erfassung des Seelischen durch ein IT-System könnte zur Entpersönlichung führen, die Person könnte hinter einem Verhaltensdatensatz verschwinden. Die aus dem Datensatz resultierenden Entscheidungen würden sich eher an den Indikatoren als am individuellen Leiden orientieren. Die Technisierung und Standardisierung von Therapieprogrammen für Zehntausende leistet der McDonaldisierung der Medizin Vorschub, bei der die Effizienz des Systems anhand interner Organisationskriterien beurteilt wird und sich nicht mehr am Nutzer orientiert [17]. Auch der Datenschutz ist ein heikles Thema: Zum einen entsteht gerade ein allgemeines Bewusstsein dafür, dass Daten im Internet in der Regel nicht sicher sind. Und es sind kaum persönlichere Daten als die zur psychischen Gesundheit und zu entsprechenden Verhaltensmustern vorstellbar. Aber selbst wenn E-Health-Programme so abgeschottet werden könnten, dass Daten nicht nach außen dringen, gibt es ein Datenschutzproblem: Häufig wird EHealth von Anbietern mit inhärenten Interessenkonflikten betrieben, z. B. von Gesundheitsanbietern und Krankenversicherungen, die sowohl den Therapieerfolg als auch eine Kostensenkung anstreben. Wer kann verhindern, dass Kostenträger anhand der Telemedizindaten „hoffnungslose Fälle“ identifizieren, denen dann bestimmte Therapien nicht mehr angeboten werden? Menschen, die sich in Telemedizinprogrammen nicht verändern, könnten in eine neue Art von Schwierigkeiten geraten. Müssen Versicherte ihre E-Health-Daten einer Lebensversicherung offenbaren, genauso wie Vorerkrankungen, und einen Risikozuschlag in Kauf nehmen, wenn die Daten auf verminderte Veränderungsfähigkeit hinweisen? Wenn ich den Notruf auslöse, wenn es mir schlecht geht, weist das auf meine Krankheitsschwere oder meine Therapiemotivation hin? Schon bietet eine Autoversicherung eine App an, die Fahrverhalten mit Ort und Uhrzeit misst und entsprechend Rabatte verspricht (http://sz.de/1.1618144). Welche Möglichkeiten eröffnen sich da für Versicherungen, die detaillierte Daten zum individuellen Gesundheitsverhalten haben? Hier werden Themen der individuellen Freiheit direkt berührt. Wenn E-Health-Systeme als Dauerprogramme angelegt werden, lassen sie die Patienten vielleicht nicht mehr los. Auf die institutionelle Chronifizierung könnte die virtuelle folgen. Die Systeme müssen flexibel sein und es ermöglichen, sie zu verlassen. Hier wird die Überprüfung des individuellen Verlaufs durch Therapeuten unumgänglich sein. Beinhaltet E-Health auch offene Diskussionskanäle, müssen Qualität und Seriosität von Informationen transparent gemacht werden [8]. Ältere Patienten sind häufig 
von Internet und Smartphones ausgeschlossen. Dass die Marktmacht der Älteren es ermöglicht, adäquate Kommunikationsmittel zu produzieren, ist erst einmal ein frommer Wunsch.

\section{Beginn einer neuen Ära?}

Steht E-Health in der Reihe der bedeutenden Innovationen der Psychiatrie im letzten Jahrhundert (Psychiatrie-Enquete, Medikamente, Psychotherapie) [18]? Wird sie einer der Treiber des Fortschritts, insbesondere vor dem Hintergrund, dass die Innovationen der Psychopharmaka derzeit in der Krise sind [19]? Welche neue Varianten (whatsapp, Facebook) kommen mit immer neuen kommunikativen Möglichkeiten?

Die E-Health ist in der Pionierphase, Lauber [20] wies in dieser Zeitschrift darauf hin, dass innovative Modellversuche immer unter bestimmten günstigen Bedingungen ausprobiert werden, und zwar mit „neu gebildeten, hoch motivierten und „von der Sache" überzeugten Teams, bisweilen mit charismatischen Führungsfiguren an der Spitze“. Der Wirksamkeitsnachweis im Alltag steht aus. Ein erster Schritt zur Akzeptanz in der Medizin ist durch Aufnahme in die S3-Leitlinie Psychosoziale Therapien getan. Es wird interessant sein zu sehen, ob bald nicht nur ein Medikament und eine Psychotherapie empfohlen werden, sondern auch eine helpline, oder ob das Vertrauen in die Wirksamkeit zu gering sein wird. Die erwähnten Nebenwirkungen werden zunächst möglicherweise subtil und unmerklich sein, aber mit der steigenden Verbreitung von E-Health an Relevanz gewinnen. Notwendig ist ein Bewusstsein für die spezifischen Risiken neuer Kommunikationstechniken. E-Health kann immer nur Mittel sein, und nicht Zweck.

\section{Literatur}

1 Bauer S, Kordy H. E-Mental-Health. Neue Medien in der psychosozialen Versorgung. Heidelberg: Springer; 2008

2 Fjeldsoe BS, Marshall AL, Miller YD. Behavior change interventions delivered by mobile telephone short-message service. Am J Prev Med 2009; 36: 165 - 173

3 Free C, Knight R, Robertson $S$ et al. Smoking cessation support delivered via mobile phone text messaging (txt2stop): a single-blind, randomised trial. Lancet 2011; 378: 49-55
4 Meyer C, Ulbricht S, Gross B et al. Adoption, reach and effectiveness of computer-based, practitioner delivered and combined smoking interventions in general medical practices: a three-arm cluster randomized trial. Drug Alcohol Depend 2012; 121: 124 - 132

5 Kächele H. Der Therapeut im Internet: Nur noch ein „human companion"? In: Bauer S, Kordy H, Hrsg. E-Mental-Health Neue Medien in der psychosozialen Versorgung. Heidelberg: Springer; 2008: 304-312

6 Wampold BE. Humanism as a common factor in psychotherapy. Psychotherapy (Chic) 2012; 49: 445 -449

7 Schomerus G. Steine auf dem Weg - Stigma und Hilfesuchverhalten. Psychiat Prax 2009; 36: 53-54

8 DGPPN. S3-Praxisleitlinien in Psychiatrie und Psychotherapie. S3-Leitlinie Psychosoziale Therapien bei schweren psychischen Erkrankungen. Heidelberg: Springer; 2013

9 Wilkinson R, Pickett K. The Spirit Level: Why Equality is Better for Everyone. London: Penguin Books; 2010

10 Mercer SW, Guthrie B, Furler J et al. Multimorbidity and the inverse care law in primary care. BMJ 2012; 344: e4152

11 Hart JT. The inverse care law. Lancet 1971; 1: 405-412

12 McLellan AT, McKay JR, Forman R et al. Reconsidering the evaluation of addiction treatment: from retrospective follow-up to concurrent recovery monitoring. Addiction 2005; 100: $447-458$

13 Ehrenreich $H$, Mangholz A, Schmitt $M$ et al. OLITA: an alternative in the treatment of therapy-resistant chronic alcoholics. First evaluation of a new approach. Eur Arch Psychiatry Clin Neurosci 1997; 247: 51 - 54

$14 \mathrm{Rehm}$ J, Shield KD. Alcohol consumption, alcohol dependence and attributable burden of disease in Europe. Potential gains from effective interventions for alcohol dependence. Toronto: Centre for Addiction and Mental Health (CAMH); 2012

15 Moser T. Psychotherapie: Wider den Beschleunigungswahn. Dtsch Arztebl Int 2012; 109: 2180

16 Suffoletto B, Callaway C, Kristan J et al. Text-message-based drinking assessments and brief interventions for young adults discharged from the emergency department. Alcohol Clin Exp Res 2012; 36: 552 - 560

17 Brüsemeister T. Die Gesellschaft als organisierte Erwartungs-Enttäuschungs-Spirale - George Ritzers These der McDonaldisierung. In: Schimank U, Volkmann U, Hrsg. Soziologische Gegenwartsdiagnosen I - Eine Bestandsaufnahme. Opladen: Leske \& Budrich/UTB; 2000: 275 289

18 Priebe S. Wo ist der Fortschritt? Psychiat Prax 2012; 39: 55- 56

19 Cressey D. Psychopharmacology in a crisis. Im Internet: 2011: http:// www.nature.com/news/2011/110614/full/news.2011.367.html (Stand: 28.06.2013)

20 Lauber C. Krankenversorgung - Versorgungsplanung - Versorgungspolitik - Versorgungsforschung. Psychiat Prax 2012; 39: 1 -2 